\title{
Epigenetic programming of adipose-derived stem cells in low birthweight individuals
}

\author{
Christa Broholm $^{1}$ - Anders H. Olsson ${ }^{1} \cdot$ Alexander Perfilyev $^{2} \cdot$ Ninna S. Hansen ${ }^{1,3}$ • \\ Maren Schrölkamp $^{1}$ - Klaudia S. Strasko ${ }^{1}$. Camilla Scheele ${ }^{3,4}$. \\ Rasmus Ribel-Madsen $^{1,5}$ • Brynjulf Mortensen ${ }^{6}$ • Sine W. Jørgensen ${ }^{6}$ - Charlotte Ling ${ }^{2}$. \\ Allan Vaag ${ }^{1,3,6}$
}

Received: 17 May 2016/Accepted: 9 August 2016 /Published online: 14 September 2016

(C) Springer-Verlag Berlin Heidelberg 2016

\begin{abstract}
Aims/hypothesis Low birthweight (LBW) is associated with dysfunctions of adipose tissue and metabolic disease in adult life. We hypothesised that altered epigenetic and transcriptional regulation of adipose-derived stem cells (ADSCs) could play a role in programming adipose tissue dysfunction in LBW individuals.

Methods ADSCs were isolated from the subcutaneous adipose tissue of 13 normal birthweight (NBW) and 13 LBW adult men. The adipocytes were cultured in vitro, and genome-wide differences in RNA expression and DNA methylation profiles were analysed in ADSCs and differentiated adipocytes.

Results We demonstrated that ADSCs from LBW individuals exhibit multiple expression changes as well as genome-wide alterations in methylation pattern. Reduced expression of the
\end{abstract}

Electronic supplementary material The online version of this article (doi:10.1007/s00125-016-4099-9) contains peer-reviewed but unedited supplementary material, which is available to authorised users.

Christa Broholm

christa.broholm@gmail.com

1 Department of Endocrinology, Diabetes and Metabolism, Rigshospitalet - Section 7652, Tagensvej 20,

DK-2200 Copenhagen, Denmark

2 Department of Clinical Sciences, Epigenetics and Diabetes, Lund University Diabetes Centre, CRC, Malmö, Sweden

3 Faculty of Health and Medical Sciences, University of Copenhagen, Copenhagen, Denmark

4 Centre of Inflammation and Metabolism, Rigshospitalet, Copenhagen, Denmark

5 Danish Diabetes Academy, Odense, Denmark

6 Steno Diabetes Center A/S, Gentofte, Denmark transcription factor cyclin T2 encoded by CCNT2 may play a key role in orchestrating several of the gene expression changes in ADSCs from LBW individuals. Indeed, silencing of CCNT2 in human adipocytes decreased leptin secretion as well as the mRNA expression of several genes involved in adipogenesis, including MGLL, LIPE, PPARG, LEP and $A D I P O Q$. Only subtle genome-wide mRNA expression and DNA methylation changes were seen in mature cultured adipocytes from LBW individuals.

Conclusions/interpretation Epigenetic and transcriptional changes in LBW individuals are most pronounced in immature ADSCs that in turn may programme physiological characteristics of the mature adipocytes that influence the risk of metabolic diseases. Reduced expression of CCNT2 may play a key role in the developmental programming of adipose tissue.

Keywords Adipocytes - Adipogenesis - Adipose tissue · Cyclin T2 $\cdot$ Epigenetics $\cdot$ Fetal programming $\cdot$ Low birthweight $\cdot$ Metabolic disease $\cdot$ Type 2 diabetes

$\begin{array}{ll}\text { Abbreviations } \\ \text { ADSC } & \text { Adipose-derived stem cells } \\ \text { CCNT2 } & \text { Cyclin T2 } \\ \text { CDK } & \text { Cyclin-dependent kinase } \\ \text { FDR } & \text { False discovery rate } \\ \text { GO } & \text { Gene ontology } \\ \text { LBW } & \text { Low birthweight } \\ \text { NBW } & \text { Normal birthweight } \\ \text { PCA } & \text { Principal component analyses } \\ \text { RT } & \text { Reverse transcription } \\ \text { SiRNA } & \text { Small interfering RNA } \\ \text { SNP } & \text { Single nucleotide polymorphism } \\ \text { STAT2 } & \text { Signal transducer and activator of transcription } 2\end{array}$




\section{Introduction}

Environmental factors leading to inadequate intrauterine nutrition or decreased placental effectiveness can result in low birthweight (LBW) [1]. In adulthood, individuals born with LBW have different phenotypic changes including reduced lean body mass [2], higher total abdominal fat mass [3], reduced insulin secretion [4] and altered expression of insulin signalling proteins in muscle and fat $[5,6]$. Together, these changes are key determinants of an increased risk of developing cardiometabolic diseases such as type 2 diabetes [7].

Epigenetic processes are conceptually attractive mechanisms explaining how events occurring in fetal life can permanently affect tissue structure and metabolism, and thereby mediate the association between LBW and the development of type 2 diabetes in adulthood. DNA methylation patterns are copied by DNA methyltransferase 1 (DNMT1) during DNA replication [8], implying that environmentally induced perturbations that occur during early development can be transmitted through cell divisions to subsequent cell generations. Alterations in the fetal environment may therefore change the epigenome of specific cells or tissues, thereby permanently changing the structure and/or function of different organs [9-12]. Interestingly, exposure to suboptimal nutrition during fetal life leads to changes in germ cell DNA methylome in male mice [13], potentially affecting even the next generation. In addition, maternal diet during early pregnancy in humans can cause persistent changes in the blood methylome of the offspring [14].

Adipose-derived stem cells (ADSCs) originate from the mesenchymal lineage and are formed in the second trimester of pregnancy [15]. ADSCs serve to generate and regenerate adult adipose tissue through differentiation into lipid-storing and metabolically active adipocytes [16], a process that is controlled by epigenetic mechanisms [17-20]. Human ADSCs can be isolated with high purity from the stromal vascular fraction of subcutaneous fat biopsies. Importantly, compared with many other cellular lineages, the process of fat cell differentiation in vitro is considered to be fairly authentic, recapitulating most of the key features of adipogenesis in vivo, including morphology, cessation of cell growth, expression of lipogenic enzymes, sensitivity to lipogenic hormones and extensive lipid accumulation [21].

We recently demonstrated that cultured pre-adipocytes from LBW humans exhibit several functional defects associated with adipogenesis, including impaired leptin and adiponectin production and decreased expression of the genes encoding peroxisome proliferator-activated receptor gamma $(P P A R G)$ and fatty acid binding protein 4 (FABP4), together suggesting functional immaturity of the cells. The impaired leptin production was associated with increased DNA methylation of the $L E P$ promoter [22]. Based on these compelling observations, we hypothesised that LBW individuals would exhibit disproportionately more widespread DNA methylation and gene expression changes in the ADSCs, and that the methylome and transcriptome of ADSCs might be an indicator of the early-life environment potentially influencing the risk of developing cardiometabolic diseases with ageing.

\section{Methods}

Study participants Thirteen individuals born with LBW (birthweight below the 10th percentile) and 13 individuals born with normal birthweight (NBW) (birthweight in the 50th-90th percentile range) were recruited through The Danish National Birth Register. Briefly, all individuals were healthy, young, age-matched male singletons, born at term, with no family history of diabetes in two generations, with a $\mathrm{BMI}<30 \mathrm{~kg} / \mathrm{m}^{2}$ and without self-reported high physical activity level ( $>10 \mathrm{~h}$ per week). Abdominal subcutaneous adipose biopsies were obtained from the individuals using a Bergstrom biopsy needle with suction [23] after an overnight fast. Five individuals participated in a study by Jørgensen et al [24] and 21 individuals in a study by Mortensen et al [25], both of which used the same stringent inclusion criteria. The number of participants included in this study is lower than the total number of participants involved in the studies by Jørgensen and Mortensen [24, 25] only because ADSCs were not available for all participants.

Study approval All participants were given oral and written information about the experimental procedure before giving their written informed consent. The study was approved by the regional ethical committee (H-A-2009-040 and H-D-2008-127) and performed according to the Declaration of Helsinki. In all data analyses, participants were identified by number rather than by name.

Isolation and culture of adipose precursor cells Adipose precursor cells were isolated from subcutaneous biopsies. A detailed description of the cell isolation and culture procedures is given in the electronic supplementary material [ESM] Methods.

Small interfering RNA transfection in adipocytes Transient transfections of human adipocytes were performed using pools of small interfering RNA (siRNA) oligos specifically targeting four different sites of STAT2 or CCNT2 mRNA (On Target Plus) to minimise any off-target effects (catalogue no. L-003221-00-0010 and L-012064-00-0010 10, Dharmacon, CO, USA). Control cells were treated with a commercial non-targeting siRNA control (D-001810-10-20; Dharmacon). Transfections were performed in differentiating adipocytes at 
day 6 using $50 \mathrm{nmol} / 1$ of siRNA and Lipofectamine RNAiMAX (catalogue no. 13778-075, Invitrogen, Carlsbad, CA, USA) in antibiotic-free differentiation media (see ESM). RNA and media were harvested at day 9 (72 $\mathrm{h}$ after transfection) and at day 12 (144 $\mathrm{h}$ after transfection). Intracellular lipid droplets were stained with Oil Red O at day 12 as previously described [22]. Leptin concentrations were measured in cell media at day 12 using Meso Scale Discovery plates (catalogue no. K151BYC-1; MSD, MD, USA) according to the manufacturer's instructions (without diluting the media samples).

Gene expression and DNA methylation arrays Genome-wide gene expression analysis was studied using the HumanHT-12 v4 Expression BeadChip (Illumina, CA, USA). Genome-wide DNA methylation was studied using the Infinium HumanMethylation450 BeadChip (Illumina). A detailed description of data processing as well as gene ontology (GO) analysis and molecular interaction networks is given in ESM Methods.

cDNA synthesis and quantitative real-time PCR Reverse transcription (RT) reactions were performed using random hexamers on $0.25 \mu \mathrm{g}$ RNA employing the High-Capacity cDNA RT kit (Applied Biosystems, CA, USA). Primers were designed using human specific databases (Ensembl) and the Universal Probe Library Assay Design Centre (https://lifescience.roche.com, accessed July 2016). The primer sequences are given in ESM Table 1. The primers were synthesised by DNA Technology (Risskov, Denmark). The real-time PCR was performed using the SYBR green method with quantification by standard curves on a ViiA 7 Real-Time PCR System (Applied Biosystems). To adjust for variations in the amount of cDNA, each gene expression quantity was normalised to that of $18 \mathrm{~S}$ ribosomal RNA.

Statistical analysis Results are presented as mean $\pm \mathrm{SD}$, mean $\pm \mathrm{SEM}$, mean differences and/or fold change. Differences in gene expression and DNA methylation between the LBW and NBW groups in ADSCs and mature adipocytes were analysed using non-paired, non-parametric tests (Wilcoxon rank-sum tests). The false discovery rate (FDR) [26] was applied to account for multiple testing in all the genome-wide analyses of gene expression and DNA methylation performed. The R project (www.r-project.org/; accessed February 2012) was used for statistical analyses and graphics, with additional packages provided by the Bioconductor project (www.bioconductor.org/; accessed February 2012) [27]. We performed a principal component analysis (PCA) of the genome-wide DNA methylation and gene expression data after batch correction and correlated the top principal components with birthweight, age and BMI to examine possible sources of variation in the data sets. The data used for PCA were based on uncorrected $p$ values. We used two-way ANOVA (SAS Software, version 9.1.3 (SAS Institute, Cary, NC, USA) to evaluate the effect of differentiation and group on STAT2 and CCNT2 expression. The residuals obtained from the ANOVA models were evaluated, and the model was accepted only if the residuals were normally distributed.

\section{Results}

Clinical characteristics of the human donors ADSCs were isolated from subcutaneous biopsies obtained from $13 \mathrm{NBW}$ and 13 LBW adult lean healthy men. The clinical characteristics of the ADSC donors are shown in Table 1. There was no significant difference in fasting serum insulin or plasma glucose (Table 1), or plasma triacylglycerol and cholesterol levels (data not shown) between LBW and NBW individuals $(p>0.05)$. However, the LBW men were lighter $(p<0.05)$ and had a tendency towards an increased WHR $(p=0.058)$, indicating a higher proportion of abdominal fat relative to hip fat. Genome-wide gene expression and DNA methylation levels were analysed in proliferating ADSCs (80\% confluent) and terminally differentiated adipocytes grown for 12 days in differentiation media. Statistical comparisons are indicated by the black arrows in Fig. 1a. The previously described phenotype of the LBW adipocytes includes impaired secretion of adiponectin and leptin together with decreased gene expression of FABP4 and PPARG [22]. Indeed, the same donors and cell cultures were used in the current study.

Genome-wide effects of birthweight on gene expression and DNA methylation To explore the effect of birthweight on genome-wide gene expression and DNA methylation,

Table 1 Clinical characteristic of ADSC donors

\begin{tabular}{llll}
\hline & LBW $(n=13)$ & NBW $(n=13)$ & $p$ value \\
\hline Birthweight $(\mathrm{kg})$ & $2.7 \pm 0.1$ & $3.7 \pm 0.2$ & $<0.0001$ \\
Age (years) & $22.4 \pm 1.7$ & $23.2 \pm 1.6$ & $\mathrm{NS}$ \\
Weight $(\mathrm{kg})$ & $73.9 \pm 7.3$ & $80.8 \pm 5.4$ & $<0.05$ \\
Height $(\mathrm{cm})$ & $178.0 \pm 4.1$ & $182.5 \pm 6.9$ & 0.063 \\
BMI $\left(\mathrm{kg} / \mathrm{m}^{2}\right)$ & $23.3 \pm 2.2$ & $24.3 \pm 2.0$ & $\mathrm{NS}$ \\
WHR & $0.90 \pm 0.1$ & $0.86 \pm 0.1$ & 0.058 \\
Total fat mass $(\mathrm{kg})$ & $13.2 \pm 4.0$ & $13.1 \pm 2.8$ & $\mathrm{NS}$ \\
Trunk fat mass $(\%)$ & $16.6 \pm 4.7$ & $15.4 \pm 3.5$ & $\mathrm{NS}$ \\
Lean body mass $(\mathrm{kg})$ & $58.5 \pm 4.5$ & $64.4 \pm 4.4$ & $<0.01$ \\
Fasting insulin $(\mathrm{pmol} / \mathrm{l})$ & $28.8 \pm 17.4$ & $37.3 \pm 20.5$ & $\mathrm{NS}$ \\
Fasting glucose $(\mathrm{mmol} / \mathrm{l})$ & $4.9 \pm 0.4$ & $5.1 \pm 0.2$ & $\mathrm{NS}$ \\
Fasting HbA $\mathrm{lc}(\%)$ & $5.2 \pm 0.3$ & $5.1 \pm 0.2$ & $\mathrm{NS}$ \\
Fasting HbA $\mathrm{lc}(\mathrm{mmol} / \mathrm{mol})$ & $32.8 \pm 3.1$ & $32.2 \pm 2.5$ & $\mathrm{NS}$ \\
\hline
\end{tabular}

Data are shown as mean $\pm \mathrm{SD}$ 
Fig. 1 Gene expression differences between ADSCs from LBW and NBW donors. (a) Study set-up. Genome-wide gene expression and DNA methylation profiles were analysed in ADSCs and mature adipocytes from NBW individuals and LBW individuals. Comparisons between sample groups were performed as indicated by the black arrows. (b) Hierarchical clustering heat map of 506 differentially expressed mRNA transcripts $(q<0.05)$ in ADSCs isolated from LBW vs NBW individuals. Each column represents ADSCs isolated from a single sample, and each row represents a single mRNA transcript. Expression levels are scaled to raw $z$ scores and plotted in a red to blue colour scale representing high to low expression signals. Hierarchical clustering was applied based on Euclidean distances. (c) GO biological processes based on 225 downregulated gene transcripts in LBW ADSCs. The number of significant genes out of all genes in the $\mathrm{GO}$ biological process is shown at the end of the bar. The $p$ values are Benjamini-Hochberg corrected. (d) GeneMANIA network illustrating genetic interactions (black lines) between genes showing significantly $(q<0.05)$ higher expression (red nodes) or lower expression (blue nodes) in ADSCs from LBW cells. To look for transcription factors (TFs) among the differentially expressed genes, the network is extended with TF targets from the ENCODE project. $\mathrm{TF}$-gene or $\mathrm{TF}-\mathrm{TF}$ interactions are indicated in the extended network as proximal (yellow lines) or distal (purple lines) TF regulation. TF targets can be assigned either as a significant differentially expressed gene in our data set (red to blue nodes), or any other gene (purple nodes) or TF (turquoise nodes) not showing differential expression in ADSCs between the two birthweight groups.

Magnified genes (CCNT2 and STAT2) are TFs with a central role in the gene interaction network a

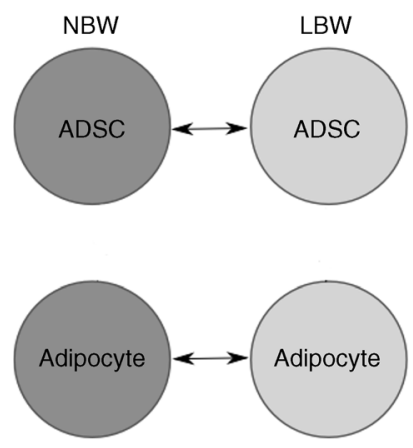

b

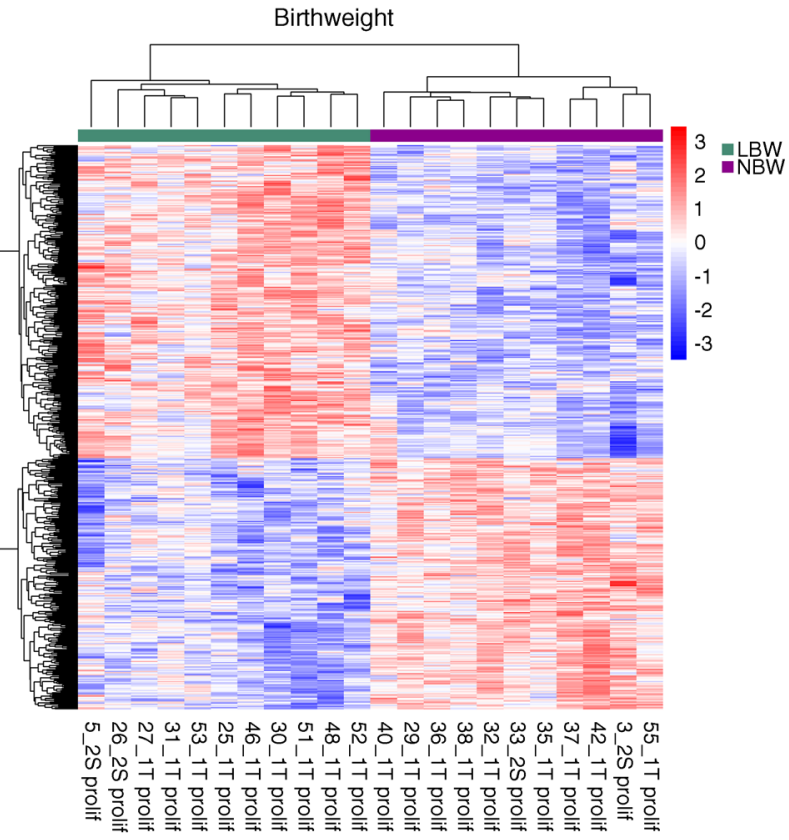

C

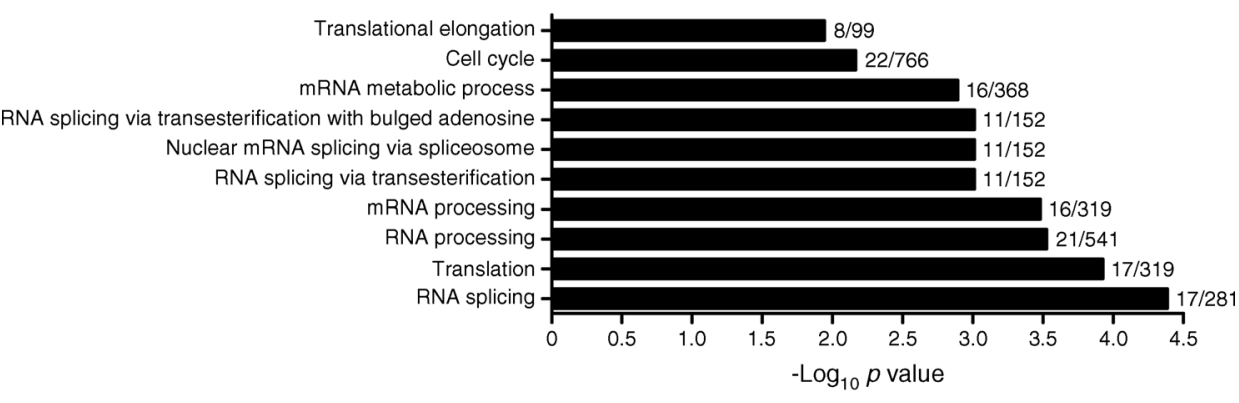

d

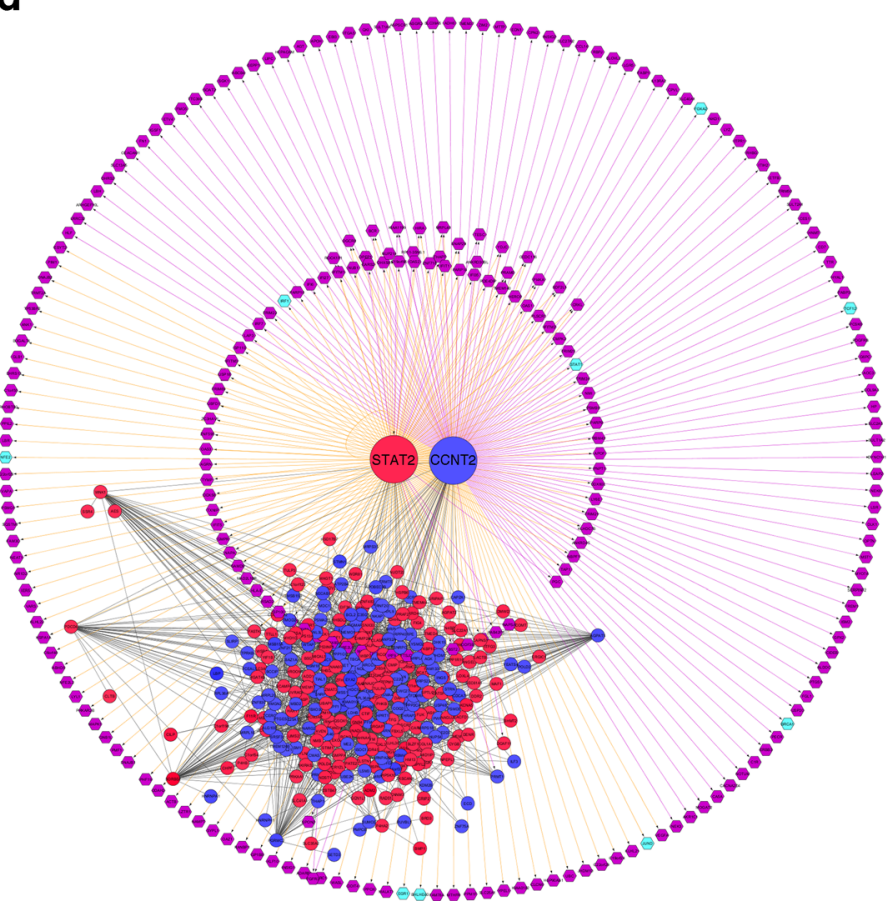


PCAs were performed first within the group of ADSCs, and second within the group of differentiated adipocytes. A significant correlation was observed between the second principal component and birthweight when analysing gene expression profiles in ADSCs from the two groups $(p=0.0234)$, whereas there was no correlation between birthweight and principal components in the differentiated adipocytes. Remarkably, a similar and even more pronounced pattern was reflected in the genome-wide methylation data, where a significant correlation was observed between the first principal component and birthweight in ADSCs $(p=0.0231)$. Again, there was no effect of birthweight on the methylation pattern in the differentiated adipocytes, suggesting that both gene expression and DNA methylation changes in LBW participants are most pronounced in immature tissue progenitor cells.

Identification of $C C N T 2$ and $S T A T 2$ mRNA as differentially expressed transcription factors in LBW ADSCs At an individual gene level, 506 Illumina-assigned gene transcripts were significantly differentially expressed at an FDR of less than $5 \%(q<0.05)$ when comparing ADSCs from the two birthweight groups (Fig. 1b, ESM Table 2). Of the differently expressed genes, 281 transcripts were upregulated in LBW ADSCs, and 225 transcripts were downregulated in LBW ADSCs. When subjecting genes downregulated in ADSCs
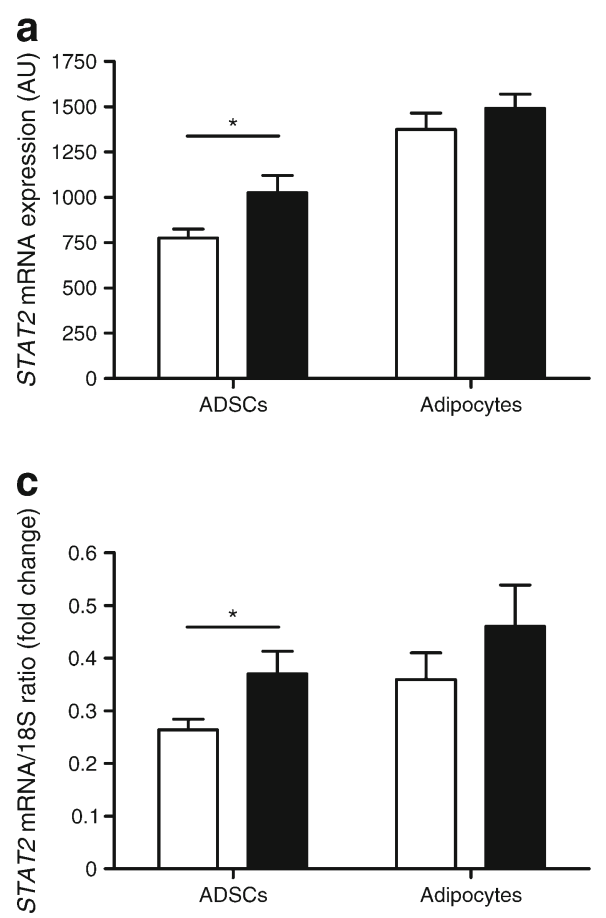

Fig. 2 (a, b) Gene expression of STAT2 and CCNT2 in ADSCs and adipocytes. Illumina mRNA expression of STAT2 (a) and CCNT2 (b) in ADSCs and differentiated adipocytes. White bars, NBW; black bars, LBW. Whereas $\log _{2}$ intensity values after robust multiarray average data processing and normalisation were used for all statistical tests, unlogged expression values are plotted in the figure, in arbitrary units (AU). Data derived from LBW individuals to GO analysis, significant enrichment of genes in ten biological processes was found. The biological processes reflected altered function of the ribosome and spliceosome, and the levels of several genes important for cell cycle progression were decreased (Fig. 1c). No significant biological processes were obtained after subjecting the upregulated transcripts in LBW ADSCs to GO analysis. To identify central transcription factors, which could drive the altered gene expression in LBW ADSCs, information from the ENCODE project (www.genome. gov/10005107/encode-project/, accessed January 2015) was applied to a gene-gene interaction network build upon genes both upregulated and downregulated in LBW ADSCs. Two differentially expressed transcription factors cyclin $\mathrm{T} 2$ (CCNT2) (downregulated in LBW) and signal transducer and activator of transcription 2 (STAT2) (upregulated in LBW) were shown to interact with a large number of genes also significantly differentially expressed in our data set (red and blue nodes) (Fig. 1d). The analysis suggested that CCNT2 and STAT2 could be central to the gene expression changes in LBW ADSCs by potentially regulating the gene expression levels, and we therefore decided to investigate CCNT2 and STAT2 in more detail. STAT2 expression increased with differentiation (ANOVA; $p<0.0001$ ) and CCNT2 expression decreased with differentiation (ANOVA, $p<0.01$ ). In addition, both STAT2 and CCNT2 expression levels were
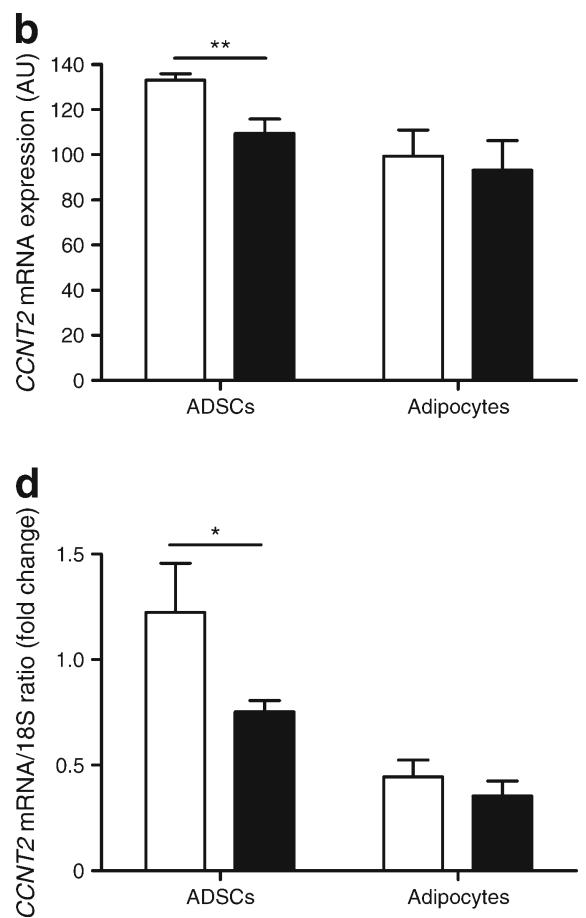

are shown as mean \pm SEM. (c, d) Validation of STAT2 (c) and CCNT2 (d) mRNA expression in ADSCs and differentiated adipocytes from NBW $(n=13)$ and LBW $(n=13)$ individuals using real-time PCR. The mRNA levels were normalised to $18 \mathrm{~S}$ ribosomal RNA. White bars, NBW; black bars, LBW. Data are shown as mean \pm SEM. The effect of differentiation was estimated using two-way ANOVA. $* p<0.05, * * p<0.01$ 
different between the birthweight groups in ADCSs but not in adipocytes (Fig. 2a, b). The differential expression was technically validated with quantitative real-time PCR, which confirmed the birthweight-related differences at the ADSC stage (Fig. 2c, d).

We found no individual $\mathrm{CpG}$ site with differential DNA methylation after FDR correction $(q<0.05)$ when comparing ADSCs between birthweight groups (ESM Table 3). The 20 most significant $\mathrm{CpG}$ sites with differential methylation between LBW and NBW ADSCs without correction for multiple testing are shown in Table 2. There was no overlap between the 20 most significant $\mathrm{CpG}$ sites and the 506 differentially expressed transcripts between NBW and LBW ADSCs. Taken together, these findings suggest that inherent DNA methylation changes in LBW ADCSs do not directly explain the birthweight-dependent alterations in CCNT2 and STAT2 expression.

$C C N T 2$ and $S T A T 2$ play a role in human adipogenesis To further investigate the role of CCNT2 and STAT2 in adipogenesis, we used siRNA to silence CCNT2 and STAT2 during early adipogenesis (day 6) in cells from five different NBW donors. Both STAT2 and CCNT2 mRNA levels were efficiently decreased after 3 days (at day 9) and after 6 days (day 12) of silencing (Fig. 3a, b). Although no differences were observed for fat accumulation (Fig. 3c), a striking decrease in the gene expression of five out of six a priori selected adipogenic genes was observed (Fig. 3d) after silencing of CCNT2. Four of the genes (PPARG, FABP4, LEP and $A D I P O Q)$ were selected based on previously reported decreased expression in LBW adipocytes [22], whereas $M G L L$ (encoding monoglycerol lipase) and LIPE (encoding hormonesensitive lipase) were selected on the basis of their important role in lipolysis. CCNT2 silencing reduced the expression of $M G L L$, LIPE, PPARG, LEP and ADIPOQ by approximately $50 \%$ compared with control cells, whereas STAT2 silencing reduced the expression of FABP 4 by more than $50 \%$. We further investigated the secretion of leptin in the media of mature adipocytes. After siRNA silencing of STAT2, leptin was not significantly altered $(p=0.1)$ (Fig. 3e); however, a modest but significant decrease was observed after siRNA silencing of CCNT2 (Fig. 3f).

We further sought to investigate whether single nucleotide polymorphisms (SNPs) in the STAT2 (12q13.3) or the CCNT2 (2q21.3) gene regions (NCBI Variation Viewer, www.ncbi. nlm.nih.gov/variation/view; accessed July 2016) were associated with cardiometabolic disease or related quantitative traits using the publicly available genome-wide association study meta-analysis results from the DIAGRAM (DIAbetes Genetics Replication And Meta-analysis) [28], CARDIoGRAM (Coronary ARtery DIsease Genome wide
Table 2 Methylation differences between NBW and LBW ADSCs

\begin{tabular}{lllll}
\hline Gene name & Probe ID & $p$ values & $q$ values & Difference (NBW minus LBW) (\%) \\
\hline $\mathrm{n} / \mathrm{a}$ & $\operatorname{cg} 14459772$ & $3.85 \times 10^{-7}$ & 0.17 & -7.5 \\
SHANK2 & $\operatorname{cg} 20170028$ & $1.35 \times 10^{-6}$ & 0.20 & -1.3 \\
LY6H & $\operatorname{cg} 09528449$ & $2.31 \times 10^{-6}$ & 0.20 & 2.9 \\
SNX14 & $\operatorname{cg} 13188409$ & $3.65 \times 10^{-6}$ & 0.20 & 1.2 \\
SLC16A11 & $\operatorname{cg} 18530716$ & $3.65 \times 10^{-6}$ & 0.20 & -2.0 \\
NCLN & $\operatorname{cg} 20667124$ & $3.65 \times 10^{-6}$ & 0.20 & -2.8 \\
AAAS & $\operatorname{cg} 23032316$ & $3.65 \times 10^{-6}$ & 0.20 & -4.3 \\
CARD11 & $\operatorname{cg} 26937500$ & $3.65 \times 10^{-6}$ & 0.20 & -1.7 \\
DMAP1 & $\operatorname{cg} 03726147$ & $5.77 \times 10^{-6}$ & 0.21 & -6.7 \\
CEACAM19 & $\operatorname{cg} 10143883$ & $5.77 \times 10^{-6}$ & 0.21 & -3.7 \\
EIF4A3 & $\operatorname{cg} 10503854$ & $5.77 \times 10^{-6}$ & 0.21 & -0.3 \\
DYNLT1 & $\operatorname{cg} 13598865$ & $5.77 \times 10^{-6}$ & 0.21 & 0.6 \\
CSK & $\operatorname{cg} 00516515$ & $8.65 \times 10^{-6}$ & 0.21 & -4.3 \\
FAM120B & $\operatorname{cg} 01956781$ & $8.65 \times 10^{-6}$ & 0.21 & 0.7 \\
n/a & $\operatorname{cg} 04594598$ & $8.65 \times 10^{-6}$ & 0.21 & 2.6 \\
n/a & $\operatorname{cg} 05759166$ & $8.65 \times 10^{-6}$ & 0.21 & -7.0 \\
BICC1 & $\operatorname{cg} 22641201$ & $8.65 \times 10^{-6}$ & 0.21 & 1.5 \\
ACOX3 & $\operatorname{cg} 22777162$ & $8.65 \times 10^{-6}$ & 0.21 & -2.2 \\
C2CD2L & $\operatorname{cg} 03947203$ & $1.29 \times 10^{-5}$ & 0.23 & -9.7 \\
TRIM11 & $\operatorname{cg} 04632980$ & $1.29 \times 10^{-5}$ & 0.23 & -5.8 \\
\hline
\end{tabular}

The 20 CpG sites with the lowest $p$ values for the methylation difference between the LBW and NBW groups are shown for ADSCs. Each CpG site is identified with its Illumina probe target ID. The difference in methylation percentage is shown as calculated by methylation in the NBW group minus methylation in the LBW group $\mathrm{n} / \mathrm{a}$, this probe has not been associated with any gene by Illumina 

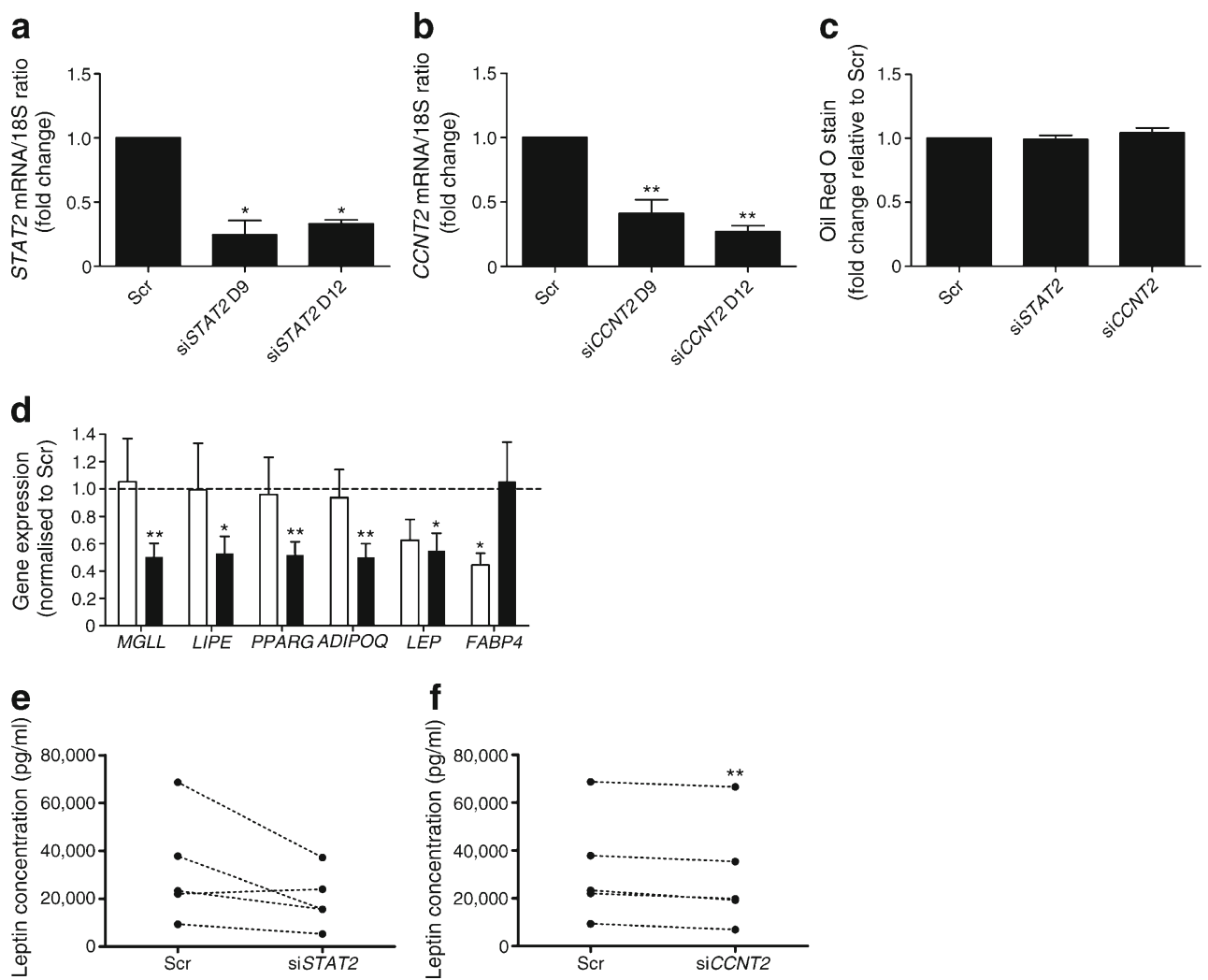

Fig. 3 Effect of STAT2 and CCNT2 on adipogenesis. (a, b) siRNA silencing of STAT2 (a) and CCNT2 (b) was performed during early differentiation (day 6) in cells isolated from five different individuals (NBW) (Scr, scrambled). Efficient knockdown of both transcripts was observed at day 9 and day 12. The mRNA levels were normalised to $18 \mathrm{~S}$ ribosomal RNA $(n=5)$. (c) Oil Red O incorporation was estimated in mature adipocytes. (d) The mRNA levels of a selected panel of

Replication and Meta-analysis) [29], MAGIC (Meta-Analyses of Glucose and Insulin-related traits Consortium) [30-32] and GIANT (Genetic Investigation of ANthropometric Traits) [33, 34] consortia. The traits examined were type 2 diabetes (case-control), coronary artery disease (case-control), fasting glucose, fasting insulin, $2 \mathrm{~h}$ glucose, HOMA-IR, BMI and WHR. The findings were reported with the original consortia $p$ values, and in order to correct for the multiple look-ups, we calculated a Bonferroni significance threshold as $p<0.05 /$ number of SNPs examined/number of traits examined. Twenty-nine SNPs were examined near STAT2 and 86 near CCNT2, giving a Bonferroni threshold of approximately $p<7 \times 10^{-5}$ to $p<2 \times 10^{-4}$ depending on the gene region. Strikingly, we found that the majority of SNPs examined in the CCNT2 region (2q21.3) were associated with BMI (59 of 85 SNPs), and one of these associations (rs1530559, $p=2 \times 10^{-6}$ ) remained significant after correction for multiple comparisons. In addition, 31 of 83 SNPs in the CCNT2 region were associated with HOMA-IR, although not significantly after correction for multiple comparisons (ESM Table 4; see ESM Table 4 legend for details). In the gene region surrounding STAT2, only a few SNPs associated nominally with the traits examined. Most interesting was the finding of four SNPs adipogenic markers were investigated in mature adipocytes. The mRNA levels were normalised to $18 \mathrm{~S}$ ribosomal RNA. White bars, siSTAT2, black bars, siCCNT2. In (a-d), paired $t$ tests were performed on raw values, whereas the figures show the ratio of the knockdown in relation to Scr (to visualise the effect of siRNA silencing as a fold change). (e, f) The amount of leptin in the media from mature adipocytes (day 12) was analysed by ELISA. $* p<0.05, * * p<0.01$

associating with coronary artery disease $(p<0.05)$ out of the eight SNPs examined in this meta-analysis (ESM Table 5; see ESM Table 5 legend for details).

A lack of genome-wide DNA methylation and gene expression changes in mature adipocytes We found no gene expression probes with altered expression or individual $\mathrm{CpG}$ sites with differential DNA methylation when comparing mature adipocytes between birthweight groups, after correction for multiple testing (ESM Tables 6 and 7). The 20 most significant $\mathrm{CpG}$ sites with differential methylation between LBW and NBW adipocytes without correction for multiple testing are shown in Table 3.

\section{Discussion}

We demonstrated that adipogenic progenitor cells from the abdominal subcutaneous fat depot of LBW individuals have an altered expression of 506 transcripts, including increased expression of STAT2 and decreased expression of CCNT2. Silencing of $C C N T 2$ changed the expression of five selected 
Table 3 Methylation differences between NBW and LBW adipocytes

\begin{tabular}{lllll}
\hline Gene name & Probe ID & $p$ values & $q$ values & Difference (NBW minus LBW) (\%) \\
\hline ARHGEF18 & $\operatorname{cg} 01977519$ & $2.31 \times 10^{-6}$ & 0.26 & 1.5 \\
FAM81B & $\operatorname{cg} 23360388$ & $2.31 \times 10^{-6}$ & 0.26 & 3.6 \\
OXCT1 & $\operatorname{cg} 21515384$ & $3.65 \times 10^{-6}$ & 0.26 & -2.8 \\
MCCC1 & $\operatorname{cg} 23476885$ & $3.65 \times 10^{-6}$ & 0.26 & -2.9 \\
n/a & $\operatorname{cg} 04568823$ & $5.77 \times 10^{-6}$ & 0.26 & 12.7 \\
n/a & $\operatorname{cg} 04850254$ & $5.77 \times 10^{-6}$ & 0.26 & 2.7 \\
SMAD9 & $\operatorname{cg} 10436026$ & $5.77 \times 10^{-6}$ & 0.26 & -8.4 \\
SPATA20 & $\operatorname{cg} 19587434$ & $5.77 \times 10^{-6}$ & 0.26 & 1.5 \\
MGRN1 & $\operatorname{cg} 01922891$ & $8.65 \times 10^{-6}$ & 0.26 & -10.2 \\
WNT5B & $\operatorname{cg} 05175896$ & $8.65 \times 10^{-6}$ & 0.26 & -12.1 \\
NUP210 & $\operatorname{cg} 08091398$ & $8.65 \times 10^{-6}$ & 0.26 & 10.2 \\
PCSK2 & $\operatorname{cg} 15060366$ & $8.65 \times 10^{-6}$ & 0.26 & 3.3 \\
ZAP70 & $\operatorname{cg} 18005337$ & $8.65 \times 10^{-6}$ & 0.26 & -14.1 \\
NLRC4 & $\operatorname{cg} 23459416$ & $8.65 \times 10^{-6}$ & 0.26 & 3.8 \\
n/a & $\operatorname{cg} 27422722$ & $8.65 \times 10^{-6}$ & 0.26 & -1.4 \\
RILP & $\operatorname{cg} 04313875$ & $1.29 \times 10^{-5}$ & 0.26 & -1.0 \\
VAX2 & $\operatorname{cg} 07094440$ & $1.29 \times 10^{-5}$ & 0.26 & 3.7 \\
n/a & $\operatorname{cg} 07617283$ & $1.29 \times 10^{-5}$ & 0.26 & 10.9 \\
SH3BP4 & $\operatorname{cg} 13858407$ & $1.29 \times 10^{-5}$ & 0.26 & -11.5 \\
C9orf40 & $\operatorname{cg} 19102271$ & $1.29 \times 10^{-5}$ & 0.26 & -1.7 \\
\hline
\end{tabular}

The $20 \mathrm{CpG}$ sites with the lowest $p$ values for the methylation difference between the LBW and NBW groups are shown for adipocytes. Each CpG site is identified with its Illumina probe target ID. The difference in methylation percentage is shown as calculated by methylation in the NBW group minus methylation in the LBW group $\mathrm{n} / \mathrm{a}$, this probe has not been associated with any gene by Illumina genes, which promote adipogenesis. CCNT2 might therefore play a role in the developmental programming of adipose tissue dysfunction in LBW humans.

Distinct epigenetic changes have been identified in the muscle and fat tissues of LBW individuals using a candidate gene approach $[9,35]$, and altered genome-wide changes in DNA methylation in the muscle tissue of LBW individuals have been observed following a metabolic challenge such as overfeeding [36]. Nonetheless, the epigenetic changes reported in mature tissue (biopsies) from LBW individuals have so far been relatively modest and cannot alone explain the strong association between LBW and the development of type 2 diabetes [7]. We hypothesised that epigenetic changes resulting from an unfavourable intrauterine environment causing LBW would be detectable and potentially more pronounced in tissue progenitor cells, e.g. ADSCs from adipose tissue. In support of our hypothesis, we here provide evidence that large parts of the variation in the gene expression and DNA methylation data set are explained by birthweight, reflected by significant correlations between the second principal component and birthweight in the gene expression data, and between the first principal component and birthweight in the DNA methylation data. Importantly, after ADSCs had differentiated into mature adipocytes, the grouping by birthweight disappeared. The finding of a clearer separation of the epigenetic fingerprints between LBW and NBW individuals in the less differentiated cells support our a priori hypothesis that epigenetic changes associated with fetal programming are most pronounced, and therefore may also be more functionally important in the immature progenitor cells.

The consequences of an altered function of ADSCs could be fewer and larger adipocytes, but it could also be the production of less developed adipocytes with an altered capacity to store fat and regulate lipolysis. Indeed, young men with LBW have increased whole-body basal lipolysis [37] as well as a significantly higher proportion of abdominal fat mass relative to total fat mass [3]. Importantly, such changes are suspected to influence the risk of developing cardiometabolic diseases [38]. The lack of genome-wide findings in mature cultured adipocytes might be explained by methodological limitations as adipocytes derived by in vitro differentiation of ADSCs will be affected by the strong adipogenic cocktail with maximal inhibition of, for example, lipolysis due to the high media contents of insulin, rosiglitazone and dexamethasone; it therefore cannot truly account for the changes that could have occurred in adipocytes in vivo.

The genome-wide transcriptomic disparities between LBW and NBW ADSCs seemed to centre on an altered regulation of the transcription factor CCNT2 (downregulated in LBW 
ADSCs). CCNT2 is the regulatory subunit of the cyclindependent kinase (CDK) pair (CDK9-CCNT2), which facilitates the transition from abortive to productive elongation by phosphorylating the large subunit of RNA polymerase II [39]. CCNT2 is essential for mouse embryogenesis [40] and is involved in early spermatogenesis [41], suggesting that CCNT2 is active during the earliest developmental stages. We found that silencing of CCNT2 markedly reduced the expression of a panel of carefully selected adipogenic markers, suggesting that CCNT2 is a central regulator of adipocyte development. In support of an important role of the CCNT2-CDK9 complex in adipogenesis, it was shown that overexpression of CDK9 in 3T3L1 adipocytes increased adipogenesis by direct binding to and phosphorylation of proliferator-activated receptor gamma [42]. We previously reported a reduced expression of PPARG, ADIPOQ and LEP in cultured adipocytes derived from LBW individuals [22]. Importantly, these are the same markers that we find decreased in the current study after CCNT2 silencing. In addition, a marked decrease of the central enzymes controlling lipolysis, $M G L$ and $L I P E$, were seen after $C C N T 2$ silencing. Altogether, reduced expression of $C C N T 2$ in LBW ADSCs might lead to delayed initiation of the adipogenic programme and subsequent development of immature adipocytes.

We next sought to investigate whether SNPs in the CCNT2 gene region $(2 \mathrm{q} 21.3)$ were associated with cardiometabolic disease and related quantitative traits in previously published genome-wide association study meta-analyses. These look-ups showed that genetic variation of the CCNT2 gene region was associated with obesity and insulin resistance, providing further evidence for a functional role of $C C N T 2$ in adipose tissue. Indeed, one SNP in the proximity of CCNT2, MAP3K19 rs1530559, associated with BMI at the suggestive genome-wide significance threshold $\left(p<5 \times 10^{-6}\right)[33]$ and also relatively strongly with fasting insulin and HOMA-IR [30].

We initially hypothesised that both epigenetic and transcriptional regulation of ADSCs could play a role in programming adipose tissue dysfunctions in LBW individuals. Although in the genome-wide DNA methylation data from ADSCs, we found a significant correlation between the first principal component and birthweight, we did not identify any single $\mathrm{CpG}$ site with differential methylation between NBW and LBW ADSCs when correcting for multiple testing. This could suggest that several small changes are operating together to change gene expression in LBW ADSCs. However, this finding also allows for the possible influence of histone modifications or changes in miRNA expression as causal factors regulating the 506 differentially expressed transcripts, including CCNT2 and STAT2, in LBW ADSCs. Importantly, the biopsies were obtained in adult men, and although the groups were well-matched according to current habits, e.g. time spent training and smoking habits, as well as according to BMI, sex and age, confounding factors including environmental stimuli might have accumulated during life and could affect the methylation and expression levels.

In conclusion, we show that ADSCs derived from healthy adult LBW humans with increased risk of developing type 2 diabetes due to their lower birthweight exhibit widespread genome-wide transcription as well as DNA methylation profile changes. This provides further evidence that inadequate fetal energy supply permanently changes the epigenetic regulation of adipose tissue progenitor cells. Further research is needed to understand whether or how these findings may be translated into a more sustainable intergenerational prevention of type 2 diabetes and associated cardiometabolic diseases in future generations.

Acknowledgements Some of the data were presented as an oral presentation at the 75th ADA scientific sessions in 2015 and at the EASD in Stockholm 2015.

Funding Support has been provided by the Danish Council for Independent Research, the Novo Nordisk Foundation, the European Foundation for the Study of Diabetes, Rigshospitalet, the Aase and Ejnar Danielsens Foundation, the Danish Diabetes Academy supported by the Novo Nordisk Foundation, and the Danish Council for Independent Research and FP7 Marie Curie Actions - COFUND: DFF-1333-00211.

Duality of interest The authors declare that there is no duality of interest associated with this manuscript.

Contribution statement $\mathrm{CB}, \mathrm{AHO}, \mathrm{AV}, \mathrm{CS}$ and $\mathrm{CL}$ conceived and designed the study, analysed the data, and wrote the paper. CB, KSS, MS and NSH carried out cell studies. AP, AHO and RR-M conducted the bioinformatics analyses. SWJ and BM collected the human cohorts and analysed and interpreted the human clinical data. All co-authors revised the manuscript and approved the final version. CB is responsible for the integrity of the work as a whole.

\section{References}

1. Wollmann HA (1998) Intrauterine growth restriction: definition and etiology. Horm Res 49(Suppl 2):1-6

2. Gale CR, Martyn CN, Kellingray S, Eastell R, Cooper C (2001) Intrauterine programming of adult body composition. J Clin Endocrinol Metab 86:267-272

3. Rasmussen EL, Malis C, Jensen CB et al (2005) Altered fat tissue distribution in young adult men who had low birth weight. Diabetes Care 28:151-153

4. Jensen CB, Storgaard H, Dela F, Holst JJ, Madsbad S, Vaag AA (2002) Early differential defects of insulin secretion and action in 19-year-old Caucasian men who had low birth weight. Diabetes 51: $1271-1280$

5. Ozanne SE, Jensen CB, Tingey KJ, Storgaard H, Madsbad S, Vaag AA (2005) Low birthweight is associated with specific changes in muscle insulin-signalling protein expression. Diabetologia 48:547552 
6. Ozanne SE, Jensen CB, Tingey KJ et al (2006) Decreased protein levels of key insulin signalling molecules in adipose tissue from young men with a low birthweight: potential link to increased risk of diabetes? Diabetologia 49:2993-2999

7. Whincup PH, Kaye SJ, Owen CG et al (2008) Birth weight and risk of type 2 diabetes: a systematic review. JAMA 300:2886-2897

8. Holliday R, Pugh JE (1975) DNA modification mechanisms and gene activity during development. Science 187:226-232

9. Brons C, Jacobsen S, Nilsson E et al (2010) Deoxyribonucleic acid methylation and gene expression of PPARGC1A in human muscle is influenced by high-fat overfeeding in a birth-weight-dependent manner. J Clin Endocrinol Metab 95:3048-3056

10. Holness MJ, Sugden MC (2006) Epigenetic regulation of metabolism in children born small for gestational age. Curr Opin Clin Nutr Metab Care 9:482-488

11. Sandovici I, Smith NH, Nitert MD et al (2011) Maternal diet and aging alter the epigenetic control of a promoter-enhancer interaction at the Hnf4a gene in rat pancreatic islets. Proc Natl Acad Sci 108: $5449-5454$

12. Gluckman PD (2012) Epigenetics and metabolism in 2011: epigenetics, the life-course and metabolic disease. Nat Rev Endocrinol 8: 74-76

13. Radford EJ, Ito $\mathrm{M}$, Shi $\mathrm{H}$ et al (2014) In utero effects. In utero undernourishment perturbs the adult sperm methylome and intergenerational metabolism. Science 345:1255903

14. Dominguez-Salas P, Moore SE, Baker MS et al (2014) Maternal nutrition at conception modulates DNA methylation of human metastable epialleles. Nat Commun 5:3746

15. Leff T, Granneman JG (2010) Adipose tissue in health and disease. Wiley, Weinheim

16. Laharrague P, Casteilla L (2010) The emergence of adipocytes. Endocr Dev 19:21-30

17. Collas $\mathrm{P}$ (2010) Programming differentiation potential in mesenchymal stem cells. Epigenetics 5:476-482

18. Li HX, Xiao L, Wang C, Gao JL, Zhai YG (2010) Review: epigenetic regulation of adipocyte differentiation and adipogenesis. J Zhejiang Univ Sci B 11:784-791

19. Sakamoto H, Kogo Y, Ohgane J et al (2008) Sequential changes in genome-wide DNA methylation status during adipocyte differentiation. Biochem Biophys Res Commun 366:360-366

20. Zhu JG, Xia L, Ji CB et al (2012) Differential DNA methylation status between human preadipocytes and mature adipocytes. Cell Biochem Biophys 63:1-15

21. Rosen ED, Spiegelman BM (2000) Molecular regulation of adipogenesis. Annu Rev Cell Dev Biol 16:145-171

22. Schultz NS, Broholm C, Gillberg L et al (2014) Impaired leptin gene expression and release in cultured preadipocytes isolated from individuals born with low birth weight. Diabetes 63:111-121

23. Bergstrom J (1975) Percutaneous needle biopsy of skeletal muscle in physiological and clinical research. Scand. Scand J Clin Lab Invest 35:609-616

24. Jørgensen SW, Brons C, Bluck L et al (2015) Metabolic response to 36 hours of fasting in young men born small vs appropriate for gestational age. Diabetologia 58:178-187

25. Mortensen B, Hingst JR, Frederiksen N et al (2013) Effect of birth weight and 12 weeks of exercise training on exercise-induced AMPK signaling in human skeletal muscle. Am J Physiol Endocrinol Metab 304:E1379-E1390
26. Benjamini Y, Hochberg Y (1995) Controlling the false discovery rate: a practical and powerful approach to multiple testing. J R Stat Soc Ser B Stat Methodol 57:289-300

27. Huber W, Carey VJ, Gentleman R et al (2015) Orchestrating highthroughput genomic analysis with Bioconductor. Nat Methods 12: 115-121

28. Morris AP, Voight BF, Teslovich TM et al (2012) Large-scale association analysis provides insights into the genetic architecture and pathophysiology of type 2 diabetes. Nat Genet 44:981-990

29. Deloukas P, Kanoni S, Willenborg C et al (2013) Large-scale association analysis identifies new risk loci for coronary artery disease. Nat Genet 45:25-33

30. Dupuis J, Langenberg C, Prokopenko I et al (2010) New genetic loci implicated in fasting glucose homeostasis and their impact on type 2 diabetes risk. Nat Genet 42:105-116

31. Saxena R, Hivert MF, Langenberg $C$ et al (2010) Genetic variation in GIPR influences the glucose and insulin responses to an oral glucose challenge. Nat Genet 42:142-148

32. Scott RA, Lagou V, Welch RP et al (2012) Large-scale association analyses identify new loci influencing glycemic traits and provide insight into the underlying biological pathways. Nat Genet 44:9911005

33. Locke AE, Kahali B, Berndt SI et al (2015) Genetic studies of body mass index yield new insights for obesity biology. Nature 518:197206

34. Shungin D, Winkler TW, Croteau-Chonka DC et al (2015) New genetic loci link adipose and insulin biology to body fat distribution. Nature 518:187-196

35. Gillberg L, Jacobsen SC, Ronn T, Brons C, Vaag A (2014) PPARGC1A DNA methylation in subcutaneous adipose tissue in low birth weight subjects-impact of 5 days of high-fat overfeeding. Metabolism 63:263-271

36. Jacobsen SC, Gillberg L, Bork-Jensen J et al (2014) Young men with low birthweight exhibit decreased plasticity of genome-wide muscle DNA methylation by high-fat overfeeding. Diabetologia 57: $1154-1158$

37. Alibegovic AC, Hojbjerre L, Sonne MP et al (2010) Increased rate of whole body lipolysis before and after 9 days of bed rest in healthy young men born with low birth weight. Am J Physiol Endocrinol Metab 298:E555-E564

38. Spalding KL, Arner E, Westermark PO et al (2008) Dynamics of fat cell turnover in humans. Nature 453:783-787

39. Bieniasz PD, Grdina TA, Bogerd HP, Cullen BR (1999) Analysis of the effect of natural sequence variation in Tat and in cyclin $\mathrm{T}$ on the formation and RNA binding properties of Tat-cyclin T complexes. J Virol 73:5777-5786

40. Kohoutek J, Li Q, Blazek D, Luo Z, Jiang H, Peterlin BM (2009) Cyclin T2 is essential for mouse embryogenesis. Mol Cell Biol 29: 3280-3285

41. Teng Y, Wang Y, Fu J, Cheng X, Miao S, Wang L (2011) Cyclin T2: a novel miR-15a target gene involved in early spermatogenesis. FEBS Lett 585:2493-2500

42. Iankova I, Petersen RK, Annicotte JS et al (2006) Peroxisome proliferator-activated receptor gamma recruits the positive transcription elongation factor $\mathrm{b}$ complex to activate transcription and promote adipogenesis. Mol Endocrinol 20:1494-1505 